\title{
Raciser la société : un projet administratif pour une société domingoise complexe (1760-1791)
}

Dominique Rogers

\section{(2) OpenEdition \\ Journals}

Édition électronique

URL : https://journals.openedition.org/jsa/11094

DOI : 10.4000/jsa. 11094

ISSN : 1957-7842

Éditeur

Société des américanistes

Édition imprimée

Date de publication : 5 décembre 2009

Pagination : 235-260

ISSN : 0037-9174

\section{Référence électronique}

Dominique Rogers, «Raciser la société : un projet administratif pour une société domingoise complexe (1760-1791) », Journal de la Société des américanistes [En ligne], 95-2 | 2009, mis en ligne le 10 décembre 2014, consulté le 02 septembre 2022. URL : http://journals.openedition.org/jsa/11094 DOl : https://doi.org/10.4000/jsa. 11094 


\title{
RACISER LA SOCIÉTÉ : UN PROJET ADMINISTRATIF POUR UNE SOCIÉTÉ DOMINGOISE COMPLEXE (1760-1791)
}

\author{
Dominique ROGERS *
}

L'auteur s'intéresse à la pertinence des termes " race ", " racisation " et " racisme " pour qualifier les processus à l'œuvre dans la société domingoise française de la fin du $\mathrm{XVIII}^{\mathrm{c}}$ siècle. Elle distingue ce qui relève des politiques mises en œuvre par les administrateurs métropolitains et les agents du pouvoir local pour lesquelles ces termes peuvent être pertinents, à certains moments (premier comité de législation), mais pas à d'autres (deuxième comité de législation). Elle leur oppose la diversité des comportements des différentes composantes de la population domingoise (Blancs, libres de couleur et esclaves) dont elle invite à approfondir l'étude. S'appuyant sur les travaux de Barbara Fields, elle fait l'hypothèse que la fin du XVIII ${ }^{\mathrm{e}}$ siècle correspond à un moment charnière de l'histoire de la colonie où l'idéologie de la race se met en place et où la société domingoise hésite sur le fonctionnement social à adopter. [Mots-clés : racialisation, lois, société, libres de couleur.]

Racializing a society : an administrative plan for a complex French colonial society (Saint-Domingue 1760-1791). The author deals with the relevance of the terms " race », " racialization » and " racism » to qualify the processes at work in the colonial society of French Saint-Domingue at the end of the xvinth century. She describes what concerns the policies implemented by the metropolitan administrators and their local agents for which these terms can be relevant at certain moments (during the first committee of legislation) but not in others (during the second one). She also takes into account the variety of the behaviour of the various members of the Domingan society (Whites, free people of colour and slaves) and she invites to deepen their study. In the same line as Barbara Fields's work, she makes the hypothesis that the end of the XVIIIth century is a turning point of the history of the colony when the ideology of race is set up and when the French Saint-Domingue society is hesitating on the social functioning to be adopted. [Key words : racialization, administration, society, free people of colour:]

"Racializar" la sociedad. Un plan administrativo para la sociedad colonial francesa compleja de Saint-Domingue (1760-1791). En este artículo se pregunta acerca de la

* Université des Antilles et de la Guyane, UFR de Lettres et Sciences Humaines, campus de Schoelcher, quartier Ravine Touza,97275 Schoelcher [dominique.rogers@wanadoo.fr].

Journal de la Société des Américanistes, 2009, 95-2, pp. 235-260. (C) Société des Américanistes. 
pertinencia de los términos « raza », « racialización » y « racismo » para calificar los procesos que se dieron en la sociedad francesa de Saint-Domingue hacia el final del siglo XVIII. Se describen las políticas implantadas por los administradores metropolitanos y los agentes del poder local en las que estas palabras pueden aplicarse en algunos momentos (primer comité legislativo) pero no en otros (segundo comité). A estas políticas se contraponen los variados comportamientos de los diferentes componentes de la población local (blancos, hombres de color libres y esclavos) que requieren ser estudiados más a fondo. En la continuidad del trabajo de Barbara Fields, se puede hacer la hipótesis que el final del siglo xviII representa una transición en la historia de la colonia en la que la ideología racial se impone, titubeando la sociedad local acerca de su modo de funcionamiento. [Palabras claves: racialización, legislación, sociedad, hombre de color libres.]

Il suffit d'avoir vécu aux Antilles quelques mois, parfois seulement quelques semaines, pour percevoir l'importance que les populations locales accordent à la couleur des individus. Jean-Luc Bonniol (2001, p. 12) a parlé à ce propos de "société marquée par la prévalence d'une identité de couleur». Alors que les travaux sur l'hémotypologie et sur la génétique des populations (Jacquart 1974; Lewontin 1974 ; Lewontin et al. 1984) ont démontré l'inadéquation du concept de «race» pour l'espèce humaine, faut-il néanmoins utiliser les notions de « race ", « racisation ", voire " racisme », pour qualifier les processus à l'œuvre dans ces sociétés? "Couleur » et "race» sont-elles irrémédiablement liées en matière de différenciation sociale ? Historienne, spécialiste des « libres de couleur $~^{1}$ de la partie française de Saint-Domingue, je considère qu'il serait intéressant de contribuer au débat en évoquant une société de la fin du XvIII ${ }^{\mathrm{c}}$ siècle présentée ordinairement ${ }^{2}$ comme la plus brutale, la plus discriminatoire et la plus raciste des sociétés esclavagistes du Nouveau Monde. L'exemple mérite d'autant plus d'attention que cette société domingoise s'est développée dans un contexte théorique complexe où la notion de "race » au sens contemporain ne se met en place que très progressivement et où les recherches les plus récentes sur les libres de couleur de cette colonie renouvellent notre connaissance de cette société. Pour traiter de la question, j'aborderai deux aspects assez différents : d'une part, un projet administratif d'organisation de la société domingoise en fonction de la couleur des individus, projet qui nous intéresse moins pour son contenu précis, déjà assez bien connu globalement, que pour sa qualification et, d'autre part, la manière dont les diverses composantes de la société locale ont appréhendé les différences que l'on désigne aujourd'hui parfois par les termes d'« ethniques » ou "raciales", mais pour lesquelles on pourrait peut-être trouver d'autres épithètes. 


\section{LE PROJET DE RÉFORME DE L'ADMINISTRATION MÉTROPOLITAINE}

Avant de tenter de qualifier ce projet de l'administration métropolitaine française, rappelons-en brièvement le contexte. Après la guerre de Sept ans (1756-1763), l'administration centrale française décide de réorganiser la société domingoise en prenant comme exemple ce qui s'est fait, bien plus tôt, en Louisiane ${ }^{3}$, dans les petites Antilles ${ }^{4}$ et en métropole ${ }^{5}$ et en légalisant le principe d'une barrière infranchissable entre les Blancs et les Noirs. Les esclaves ne sont pas la cible visée ; leur sort, ordinairement misérable, ne permet aucune confusion, même avec le plus pauvre des Blancs. La cible, ce sont les libres de couleur : des hommes noirs ou métis, libres de naissance ou par affranchissement, qui, en vertu des articles LVII et LIX du Code noir de 1685, jouissent des mêmes droits et privilèges que les Blancs. Le Code ne mentionne aucun argument pigmentaire ou racial pour justifier l'esclavage et, en accordant aux anciens esclaves l'égalité des droits avec les autres sujets du roi, il démontre une totale indifférence du législateur à une éventuelle "macule servile» ou pigmentaire. Si l'usage favorise l'affranchissement des métis, la loi ne distingue que les affranchis et les libres de naissance. L'article LVIII du Code noir obligeait les premiers à marquer un certain respect à leurs anciens maîtres, alors qu'il n'en était rien pour les seconds.

Pour tenter de mettre en place cette barrière, les administrateurs nommés par l'État et les cours locales prennent des mesures discriminatoires à l'encontre des libres de couleur de Saint-Domingue. À partir de la fin des années 1750, plusieurs arrêts limitent leur possibilité de porter des armes : interdiction de porter une machette (1758) ou une épée (1761), sauf s'ils sont en service pour la milice ou la maréchaussée (1762). Entre 1758 et 1767, il leur est aussi interdit de vendre ou d'acheter de la poudre ou des munitions sans autorisation du gouverneur. Des professions, des fonctions et des états prestigieux leur sont aussi progressivement enlevés : l'accès à la prêtrise et à la noblesse en 1763, les professions de chirurgien et de médecin en 1764, l'exercice d'une charge ou fonction publique en $1767^{6}$, le rang d'officier de milice en 1768. De 1770 à 1782, l'offensive se radicalise en s'attaquant à une population de couleur beaucoup plus large. En 1772, dans la juridiction de Port-au-Prince, les assemblées nocturnes d'hommes de couleur libres sont soumises à l'autorisation des juges. En 1773, les administrateurs interdisent aux libres ${ }^{7}$ de porter un nom de Blanc et leur imposent l'adoption d'un surnom d'origine africaine. La même année, ils édictent une loi somptuaire. Enfin, en 1783, les libres de couleur ne peuvent plus être qualifiés de sieur et de dame dans les documents officiels.

Après 1783, les règlements explicitement discriminatoires envers les libres de couleur cessent. Les nouveaux administrateurs envoyés à Saint-Domingue sont chargés de « recueillir le sentiment des conseils supérieurs, des chambres d'agriculture et des habitants qu'ils jugent les plus dignes de leur confiance » pour 
« tempérer le parti pris de la dégradation établie [contre les libres de couleur] et [de] lui donner même un terme ${ }^{8}$. En effet, à Paris, les choses ont changé. Dans les années 1760, les ministres des colonies (Praslin en 1766, Bourgeois de Boynes en $1772^{9}$ ) et le premier comité de législation considéraient les libres de couleur comme d'anciens esclaves et, à ce titre, des ennemis des Blancs. À la fin des années 1770, « les personnes les plus réfléchies 》- notamment les membres du deuxième comité de législation (1778-1781), mais aussi le maréchal de Castries (Debbasch 1967, pp. 126-130) - estiment que les libres de couleur doivent être perçus « comme la barrière la plus forte opposée à tout trouble de la part des esclaves ${ }^{10}$. Les libres de couleur apparaissent alors comme des propriétaires d'esclaves, nécessairement attachés au maintien du système. Cependant, en 1788, lorsque les instructions ministérielles annoncent la décision « de tempérer [...] la dégradation établie », le ministère choisit d'attendre «l'époque où les signes qui attestent l'origine des gens de couleur auront disparu » ${ }^{11}$. Beaucoup, à la suite de Debbasch (ibid., p. 20), en concluent que la société domingoise a « résisté victorieusement à ces pressions » métropolitaines dissidentes jusqu'à la fin de l'Ancien Régime.

Comment doit-on qualifier le processus administratif ici rappelé ? Pour Debbasch (ibid., p. 34), il s'agit de la mise en place d'un « régime de castes fondé sur la distinction des couleurs ». Cette analyse est-elle suffisante ? Ou doit-on lui préférer celle d'une tentative de « racisation » ou de « racialisation » de la société ?

À certains égards, l'expression "régime de castes » peut sembler inadaptée pour décrire la société domingoise. Celle-ci est, certes, composée de trois groupes, mais ils ne sont pas fermés à la manière d'une caste et ne se différencient pas strictement par la couleur. D'un côté, les esclaves comme les libres de couleur sont des Noirs ou des métis ; d'un autre, les esclaves par l'affranchissement deviennent des libres de couleur. Les Blancs mésalliés sont exclus de leur groupe d'origine, tandis qu'un certain nombre de métis très clairs parviennent à franchir la ligne et sont assimilés de fait aux Blancs. Néanmoins, s'il s'agit de caractériser le projet des administrateurs, l'expression "régime de castes » est pertinente car elle suggère bien la création de catégories étanches.

Faudrait-il néanmoins préférer les termes de « racisation » ou de « racialisation » ? En effet, la référence au critère de la couleur pour distinguer les populations entre elles peut sembler insuffisante pour caractériser un système dont la dimension raciste ou raciale paraît évidente. Cette référence ne légitime-t-elle pas des discriminations envers un groupe d'individus délimité par leur couleur, c'est-à-dire, dans le langage commun, en fonction d'un des critères fondamentaux définissant la notion de "race » ${ }^{12}$ ? Dans une première version de ce texte, nous avions envisagé d'employer le terme "racialisation» pour tenter de rendre compte du processus que nous voulions observer. Nous empruntions ce néologisme à Michel Wieworka (1996) qui le définit en ces termes : " processus par 
lequel une société se représente de façon plus ou moins décisive, comme constituée de groupes raciaux, de races en concurrence et en opposition ». La « racialisation » étant en fait une variété de "racisation », néologisme inventé par l'anthropologue Colette Guillaumin (2002), moins à la mode mais sans doute plus précis, il a paru plus opportun de préférer ce dernier terme. La " racisation » désigne un processus dans lequel un groupe dominant crée " des catégories ", qu'il opprime " au nom d'un signe biologique irréversible ». Dans une formulation plus scientifique, Véronique de Rudder (1998, p. 34) le décrit comme « l'attribution ou la revendication d'appartenance d'un ensemble particulier d'individus définis par un ensemble syncrétique et indissociable de caractéristiques naturelles et culturelles, physiologiques et psychologiques, biologiques et mentales ». De prime abord, la volonté des administrateurs des années 1760 de séparer strictement les populations en fonction de leur couleur et leur perception de ces composantes comme ennemies semblent participer d'une démarche d'exclusion en fonction d'un signe biologique et donc d'un processus de " racisation ».

Pourtant, ne faudrait-il pas d'abord que les populations opposées correspondent effectivement à des groupes raciaux ou que l'on peut considérer comme tels ? $\mathrm{Si}$ on analyse le discours des contemporains, on est confronté au fait que ceux-ci ne parlent jamais de préjugé de race, mais de préjugé de couleur. On le sait, le terme « race » n'a acquis que progressivement son sens moderne (Jouanna 2003). Les premières classifications de l'homo sapiens en races distinctes apparaissent avec François Bernier en 1684, puis avec l'histoire naturelle de Buffon en 1749, le Systema naturae de Linné en 1758, enfin le De generis humani varietate nativa de Johan Friedrich Blumenbach en 1776. Dès lors, on trouve les expressions de " race blanche », « race noire ou nègre » chez le vicomte de Mirabeau, Rousselot de Surgy ${ }^{13}$, chez Pierre-Victor Malouet, chez Hilliard d'Auberteuil et chez bien d'autres. L'abbé Raynal (1780, p. 292) parle de la « race vigoureuse des mulâtres », Émilien Petit de la "race indienne », tandis que monsieur de SaintLambert souhaite que l'on ne distingue aux colonies que « deux races d'hommes, celle des libres et celle des esclaves ${ }^{14}$. À l'évidence, les uns et les autres ne se réfèrent pas exactement aux mêmes choses et, en tout cas, pas à ce que l'usage commun contemporain appelle des races. Ici sont en effet juxtaposées ce que l'on nomme parfois les "catégories pures » et les "catégories métisses 》 et, plus simplement, des groupes humains, voire des classes. Utiliser alors le terme de « race » ou de « racisation » pour décrire le processus administratif proposé, ne nous ferait-il pas courir le risque d'une certaine confusion?

De manière plus fondamentale, l'argumentaire développé par les administrateurs pour justifier cette nouvelle politique suggère aussi au chercheur la prudence, car la différence alléguée entre les Noirs et ies Blancs n'est pas d'ordre essentialiste, mais simplement contingente. $\mathrm{Si}$, comme le souligne Colette Guillaumin $(2002$, p. 9), la race est une construction imaginaire, une "vérité imaginaire et non concrète » dont il n'est pas nécessaire d'établir la réalité 
physique, elle suppose néanmoins l'affirrmation d'une différence d'ordre biologique ${ }^{15}$ et pas seulement chromatique. Or les discours des propagandistes de cette politique sont loin d'être aussi radicaux. Certes, en 1771, Bourgeois de Boynes évoque une différence de nature entre les Noirs et les Blancs : «les administrateurs ne doivent pas accorder de grâce qui tendrait à réduire la différence que la nature a mise entre les Blancs et les Noirs et que le préjugé politique a eu soin d'entretenir comme une distance à laquelle les gens de couleur et leurs descendants ne devaient jamais atteindre » ${ }^{16}$. Émilien Petit (1777, p. 283), le président du premier comité de législation, parle aussi de "la supériorité du sang blanc », distinct selon lui de celui des Noirs. Néanmoins, lorsqu'il s'agit d'expliquer les conséquences funestes que peut avoir une promesse d'affranchissement, il compare l'attitude des esclaves à celles des domestiques blancs de métropole que le désir de jouir plus vite « d'un legs qui les rend à eux-mêmes, abandonnent, dans la maladie, s'ils n'en précipitent pas la fin de la vie, les maîtres assez imprudens [sic] pour faire connaître différentes dispositions qui engagent à souhaiter leur mort au lieu de veiller à leur conservation » (Petit 1777, section VI). Preuve de leur commune humanité, même pour Émilien Petit ${ }^{17}$. Le texte des instructions confiées aux administrateurs va certes plus loin en suggérant de mettre plus de distance "entre les deux espèces». Cette terminologie, à une époque où ces notions ne sont pas très claires, est-elle bien significative ? La très grande majorité des contemporains du XvIII ${ }^{\mathrm{e}}$ siècle (Blumenbach, Buffon, Linné etc.) restent foncièrement monogénistes et donc convaincus de l'unicité de l'espèce humaine, à la différence de ce que l'on observe à la fin du siècle suivant.

$\mathrm{Au} \mathrm{xVIII}^{\mathrm{e}}$ siècle, l'administration métropolitaine justifie ordinairement la subordination des hommes de couleur par une infériorité des Noirs liée à l'esclavage. En 1776, les riches libres de couleur de Gorée, invités à émigrer en Guyane après le traité de Paris, ont obtenu un statut différent ${ }^{18}$ de celui des autres hommes de couleur de la colonie. Comme le précise Sartine aux gouverneurs de la Guyane de l'époque : "C'est l'esclavage et non la couleur qui imprime aux nègres une tache ineffaçable » (Tarrade 1995, p. 139). De manière tout aussi explicite, le ministère justifie la nouvelle législation, non par un mépris épidermique, mais par la nécessité politique de maintenir les esclaves dans la sujétion pour éviter les révoltes. Les instructions envoyées à Saint-Domingue précisent :

Les gens de couleur sont libres ou esclaves, les libres sont des affranchis ou des descendants d'affranchis : à quelque distance qu'ils soient de leur origine, ils conservent toujours la tache de l'esclavage. [...] Cette distinction rigoureuse, observée même après la liberté, est le principal lien de la subordination par l'opinion qu'il en résulte que sa couleur est vouée à la servitude et que rien ne peut le rendre égal à son maître. ${ }^{19}$

N'est-ce qu'un artifice ? Le contexte historique des années 1750 et 1780 , avec la conspiration de Macandal qui a fait trembler la colonie, les traités de paix imposés aux Blancs par les Saramaka et les Ndjuka du Surinam comme cela avait 
été aussi le cas pour les Noirs marrons de la Jamaïque dès 1739, pouvait légitimement réveiller les craintes. De plus, dans le même temps, le déséquilibre entre la population blanche et la main d'œuvre servile ne cessait d'augmenter. Enfin, lorsqu'en 1788, le ministère envisage de mettre un terme au préjugé, il entend véritablement « confondre [les libres de couleur] avec les Européens et les Créoles ", preuve que les différences entre les uns et les autres ne sont pas perçues comme essentialistes, encore moins génotypiques ou simplement biologiques, et pas du tout comme irréversibles, du moins pour les réformateurs du deuxième comité de législation. Or si le critère de différenciation n'est ni biologique, ni essentialiste, ni irréversible, peut-on encore parler de race?

Ne pourrait-on chercher ailleurs et, s'inspirant de l'exemple de l'Amérique espagnole, analyser cette politique administrative comme une très classique tentative de réorganisation de la société coloniale en fonction de catégories fermées comparables à celles des sociétés d'ordres européennes ${ }^{20}$ ? Au Mexique, Federica Morelli (commentaire oral 2006) constate qu'il y a eu pendant « toute l'époque coloniale, et surtout à partir du xvII siècle, [...] une tension constante entre l'image traditionnelle de la société ordonnée et les pratiques et les dispositions sociales engendrées de facto par les conditions de la conquête et de la colonisation " .

Vers 1640, par exemple, certains curés de la ville de Mexico avaient des registres de mariage séparés pour les différentes castes. Au fur et à mesure que les processus de métissage biologique se réalisaient et que les combinaisons et transformations se multipliaient, se multipliaient également les initiatives pour décrire ces phénomènes : des taxinomies notamment qui, se fondant sur les degrés de relation et la gradation de la couleur de la peau, couvraient toute la gamme du Blanc au Noir. Les célèbres "pinturas de castas " constituent un magnifique exemple de ces initiatives : par ces peintures les artistes essaient d'illustrer un système classificatoire, conçu afin de faire l'apologie et de maintenir la suprématie sociale, d'une élite créole qui se sentait menacée par la contamination. Les efforts compliqués de ces artistes pour représenter et classer en sous-groupes familiers toutes les combinaisons, croisements raciaux et mélanges de couleurs possibles et imaginables ressemblent à une tentative d'imposer un ordre sur la confusion. Dans cette espèce de " pigmentocratie », la « blancheur » se transforma, au moins en théorie, en indicateur de la position dans l'échelle sociale. L'accusation de sang-mêlé, qui impliquait le stigmate d'illégitimité, était utilisée pour justifier une politique ségrégationniste qui excluait des castes des charges publiques et également de l'affiliation aux corporations et confréries. Cependant, comme l'ont démontré plusieurs recherches, les barrières de cette ségrégation étaient bel et bien franchissables, ce qui engendrait de fait une sorte de flexibilité ethnique légalisée. (Douglas Cope 1994)

L'État, d'ailleurs, fut contraint de le reconnaître en mettant en place des mesures de gracia al sacar permettant aux plus riches de jouir des mêmes avantages que les Espagnols, et cela quelle qu'ait été leur couleur. Comme le note Federica Morelli (commentaire oral 2006), «bien que l'Amérique coloniale 
espagnole se soit transformée en une société codifiée par la couleur, l'identification entre la couleur et le statut social n'y était pas du tout absolue pour autant ».

Cette grille d'interprétation peut s'appliquer, nous semble-t-il, de manière assez pertinente au cas domingois, même si cela exige quelques explications. Une analyse sommaire pourrait laisser croire à l'inadaptation de cette grille en alléguant que la société domingoise était, depuis le XVII ${ }^{\mathrm{e}}$ siècle, beaucoup plus fluide que la société métropolitaine et que les seules mesures de changement envisagées concernent les libres de couleur. En fait, la question est peu étudiée et la réalité est un peu différente. À la fin du XVIII ${ }^{\mathrm{e}}$ siècle, les tentatives de réorganisation sociale dans un sens plus traditionnel concernent aussi, quoique dans une moindre mesure, les «petits Blancs». Certaines instructions données aux services de police suggèrent l'existence d'un courant favorable à une hiérarchisation plus traditionnelle ${ }^{21}$ en ce qu'elles envisagent de ne pas accorder plus d'égards aux petits Blancs qu'aux libres de couleur, contrairement à l'attitude à avoir vis-à-vis des « Blancs domiciliés » ${ }^{22}$ ou des « habitants » ${ }^{23}$ suivant les textes. Les cahiers de doléances des «grands Blancs» de 1789 (Maurel 1935) qui mentionnent l'application contre eux de la "grande police » vont dans le même sens. Les difficultés d'insertion des nouveaux immigrants du XvıI ${ }^{\mathrm{e}}$ siècle, le mépris dont ils sont victimes de la part de la population blanche et les pétitions contre leur venue, l'attestent également (Cauna 1998, pp. 229-262). Les travaux de Frostin (1973), quant à eux, documentent fort bien cette peur réelle des petits Blancs d'une réorganisation sociale en fonction de la fortune et du statut, comme cela s'était pratiqué au XVII ${ }^{\mathrm{e}}$ siècle lorsque engagés blancs et esclaves noirs étaient vendus et traités au quotidien de façon également cruelle. Dès lors, le projet concurrent ${ }^{24}$, dont Émilien Petit fut l'apologiste, tendant à regrouper l'ensemble de la population blanche dans un groupe unique, lié à la France, dans le cadre d'un processus plus large de redéfinition de l'identité française, nous semble n'exprimer qu'une des solutions envisagées par la société domingoise, par ailleurs si souvent et si longtemps tentée par l'autonomie.

Un détour par la situation guyanaise permet d'enrichir le débat. Selon l'anthropologue Marie-José Jolivet (communication orale 2006), les projets d'organisation sociale visent toujours à «asseoir la hiérarchie mise en place avec la colonisation esclavagiste » et favorisent les Blancs au détriment des autres.

Il faut être Blanc, chrétien et de naissance honorable pour faire partie du groupe situé au sommet, le fait d'être esclave (indépendamment de sa place sur l'échelle chromatique) suffit à rejeter toute personne au bas de cette même échelle qui, toutefois, descend encore jusqu'au "Bossale» et au "Sauvage ». Quand on sait, par ailleurs, que le «Sauvage » est amérindien et le «Bossale» africain, on voit mieux apparaître la position de l'esclave « créole », puis du « Créole » tout court par rapport à ceux qui peuvent encore se définir par l'une ou l'autre des précédentes catégories (les deux existant en Guyane). ${ }^{25}$ 
La situation domingoise est, malgré tout, différente de la guyanaise, tout comme d'ailleurs de celle de la Nouvelle France ${ }^{26}$. À Saint-Domingue, l'administration centrale institue une hiérarchie différente. En 1767, le Roi, au travers de son ministre de la Marine, rappelle que les Indiens sont assimilés en tout aux Blancs, à la différence des Noirs et de leurs descendants.

La raison de cette différence porte sur ce que les Indiens sont nés libres et ont toujours conservé l'avantage de la liberté, dans les colonies, tandis que les nègres n'y ont été introduits que pour y demeurer dans l'état d'esclavage, première tache qui s'étend à tous leurs descendants. ${ }^{27}$

De manière très concrète, les Indiens et leurs descendants peuvent accéder à toutes les charges et fonctions publiques à Saint-Domingue ainsi qu'à la noblesse. Les arguments sont bien sûr historiquement fallacieux, puisque les Indiens ont aussi été réduits en esclavage et que les libres de Gorée ne sont pas venus comme esclaves à Saint-Domingue. Une fois de plus cependant, l'État semble construire une hiérarchie qui ne fonctionne pas selon une logique raciale simple. Pour être au sommet, on peut être Blanc, Amérindien ou descendant d'Amérindien. Dès lors, même dans cette phase aiguë de discrimination envers les libres de couleur, il n'y a pas de crispation sur la blancheur absolue. La société blanche locale entérine d'ailleurs cette logique. Lors des dénonciations pour mésalliance comme dans l'affaire Chapuizet (Debbasch 1967, pp. 71-74), l'argumentaire des accusés effectivement métis n'est pas " ma famille a toujours été blanche », mais « mon aïeule était une Indienne ». Ils utilisent sans doute la brèche ouverte par l'administration, tout en affirmant aussi leur absence de rejet de la différence. Dès lors, si le processus de hiérarchisation sociale proposé par l'État n'obéit pas à un discours idéologique unique, mais varie dans le temps et l'espace, il apparaît possible, sinon nécessaire, de suggérer qu'il s'élabore en fonction d'un contexte stratégique et/ou social particulier que l'on ne doit pas évacuer.

À ce stade, il nous semble que le projet des administrateurs des années 1760-1770, distinct de celui des années 1780 , peut sans doute être qualifié de processus de racisation de la société domingoise, par le fait même qu'il entend créer une barrière infranchissable entre les hommes en mettant en avant le critère de la couleur, même si les promoteurs de cette réforme n'ont pas les outils intellectuels pour parler en termes de race et qu'ils ont peut-être le sentiment de participer à une tentative de réorganisation beaucoup plus classique. Nous nous écartons ici du point de vue de Colette Guillaumin, pour qui la notion de " race » au sens contemporain de ce mot ne peut guère s'appliquer avant le xix ${ }^{\mathrm{e}}$ siècle. Aux États-Unis où des débats importants ont divisé les chercheurs sur cette thématique pendant de nombreuses années, nous nous situerions volontiers aux côtés de ceux qui pensent qu'il est possible de faire usage de ces termes, même s'il n'y a pas de justification biologique des différences. "The results of prejudice [seem] more important than their ideological underpinnings ${ }^{28}$ (Anderson 2005, p. 101). 
Jean-Luc Bonniol (2007) adopte d'ailleurs une posture similaire. Il faudrait néanmoins, pour en valider la pertinence, que le fonctionnement social des habitants de la colonie française de Saint-Domingue corrobore cette analyse.

\section{LA SOCIÉTÉ DOMINGOISE FACE AU PROJET MÉTROPOLITAIN}

Au-delà des projets administratifs, comment la société locale a-t-elle appréhendé sa diversité chromatique ? Pour beaucoup, la réponse est évidente, tant il est d'usage d'aflirmer que l'inspiration des règlements discriminatoires se trouve aux îles. La présence du Domingois Émilien Petit à la présidence du premier comité de législation, comme celle de Jean Dubuc au bureau des colonies, ou encore le soutien financier de l'État aux travaux de Médéric Moreau de SaintMéry en seraient une illustration évidente. Néanmoins, les travaux les plus récents ${ }^{29}$ sur les Noirs en France comme d'autres plus anciens ${ }^{30}$ suggèrent que la peur du mélange des races, ressentie par les élites françaises, explique en partie les décisions prises à une époque où les Noirs sont plus nombreux et s'intègrent assez facilement aux locaux (Boulle 2007). Pourtant, lorsqu'il s'agit d'étayer leur conviction, les historiens ont la fàcheuse habitude de n'interroger que la composante blanche de la population domingoise. Ils évoquent le silence des esclaves noirs, voire leur immobilisme total, et proclament le quarteron Julien Raimond leader incontesté des libres de couleur. Ils citent à l'envi le voyageur Alexandre Stanislas de Wimpffen, le colon métropolitain Hilliard d'Auberteuil et le juriste créole Moreau de Saint-Méry. Ils aftirment volontiers que les autres adhèrent au modèle proposé par l'administration, sauf quelques rares exceptions, toujours discrètes en public. Le discours dominant des élites exprime-t-il autre chose que sa propre perception des choses ? En diversifiant les sources d'information et en examinant les comportements des différentes composantes de la société domingoise vis-à-vis du projet métropolitain, la nature et les valeurs de cette société se révèlent d'une manière plus fine et plus exacte. Il n'est pas possible d'en rendre compte ici de manière exhaustive, mais nous en évoquerons quelques aspects.

Du côté des élites coloniales, consultées à plusieurs reprises sur la question du préjugé de couleur dans les quarante dernières années de l'Ancien Régime, nous disposons a minima d'une pluralité de témoignages différents du discours unanimiste trop souvent allégué. Lorsqu'en 1797 Moreau de Saint-Méry divise la population domingoise en treize classes d'individus, distinctes par " la nuance de la couleur de leur peau » et par différents éléments phénotypiques, il se positionne effectivement dans une logique raciale. On retrouve dans l'introduction de la Description... à la fois l'idée de la supériorité de l'homme blanc et celle de l'impossibilité d'une quelconque fusion en raison d'une différence biologique. Néanmoins, l'analyse fine de l'œuvre du juriste martiniquais montre qu'il n'est pas le théoricien raciste radical que l'on a souvent fait de lui. Dans Loix et 
constitutions..., il est fait mention de plusieurs arrêts favorables aux libres de couleur. Dans Description... de la partie française de l'isle de Saint-Domingue, maints passages laudateurs révèlent l'ambivalence du regard de Moreau de Saint-Méry (Rogers 2006). À propos du préjugé de couleur, il précise :

Cet indice, auquel il serait peut-être plus dangereux de croire, c'est l'œil du préjugé qui le voit et, s'il se promenait dans l'Europe entière, il trouverait, avec ce système, de quoi y former aussi une nomenclature colorée ; car qui n'a pas observé, en voyageant dans cette partie du monde, des teints bien obscurs et des traits qui semblent appartenir à l'Afrique ? (Moreau de Saint-Méry 1984, tome 1, p. 100)

Marcel Dorigny note, nous semble-t-il très justement : « sa vision du cloisonnement racial dans les colonies était avant tout fonctionnelle » (Dorigny, in Moreau de Saint-Méry 2004, tome 1, pp. XIX-XX). De manière encore plus caractéristique, dès les années 1770 , les élites coloniales consultées - parmi lesquelles on compte Barré de Saint-Vénant, Hilliard d'Auberteuil, Malouet d'Alibert - ne parlent que de l'assimilation des libres de couleur avec les Blancs et dépassent donc la logique raciale des administrateurs, puisqu'ils envisagent de faire abstraction de l'ascendance objective. Certes, le terme envisagé est parfois lointain et concerne essentiellement les plus clairs. Hilliard d'Auberteuil (1777, tome 2, p. 82) suggère d'attendre le sixième degré de métissage comme l'avait proposé l'amiral d'Estaing dès $1765^{31}$. En 1776, Barré de Saint-Vénant propose plutôt le stade du quarteron. Pierre Victor Malouet d'Alibert, ordonnateur de Saint-Domingue entre 1765 et 1769 , puis époux d'une Domingoise à partir de 1785, va même beaucoup plus loin (Debien et Thésée 1975). En 1785, il envisage de normaliser les relations entre les Blancs et les libres, de ne distinguer parmi ceux-ci que deux ou trois classes d'hommes en fonction, non de la couleur, mais des activités économiques et de l'accès à la propriété (Malouet 1803).

Les conseillers supérieurs et, plus largement, les praticiens du droit n'ont guère laissé d'ouvrages exposant leur position, mais les choix qu'ils ont opérés dans la mise en œuvre de la nouvelle politique nous semblent éclairants. À Saint-Domingue, les conseils supérieurs, chargés d'enregistrer les règlements de police émanant des administrateurs et d'interpréter le droit par leur fonction de haute cour, sont un rouage essentiel de la politique administrative. Or le conseil supérieur de Port-au-Prince a proposé une vision alternative de la norme administrative et s'est opposé avec fermeté aux projets discriminatoires de l'intendant intérimaire Alexandre Lebrasseur. En 1781 et 1782, il refuse d'enregistrer les trois règlements qui limitent l'accès des libres aux professions de sage-femme, d'apothicaire ${ }^{32}$ et d'orfèvre. Les conseillers argumentent : « La police laisse les gens de couleur libres jouir de la faculté de travailler [...] certainement comprise dans l'article LIX de l'édit de 1685, par lequel le roi accorde aux affranchis les mêmes droits, privilèges et immunités dont jouissent les personnes nées libres » ${ }^{33}$. Cette ordonnance est « une atteinte directe portée à l'état des affranchis en général » 
(Archives nationales, fonds Colonies, C9a 151, le 4/5/1781). "Messieurs les administrateurs se croiraient-ils autorisés à détruire la disposition d'un édit ? » (ibid.). La résistance des conseillers s'inscrit sans doute dans un conflit de pouvoir plus large, mais on remarquera qu'ils ne se sont pas engouffrés dans cette porte largement ouverte de la discrimination légale des libres de couleur. $\mathrm{Ni}$ l'ordonnance sur les orfèvres, ni celle sur les sages-femmes ne furent enregistrées et ne furent donc appliquées ${ }^{34}$. Le ministère, acquis alors aux idées nouvelles, désavoue d'ailleurs la prise d'initiative des administrateurs : « en refusant l'enregistrement d'un pareil règlement, contraire à toutes les dispositions de l'ordonnance du 30 avril 1764, le conseil de Port-au-Prince n'a fait que se conformer aux ordonnances des mois de mars 1766 et mai 1775, qui assurent irrévocablement à Saint-Domingue le maintien de l'autorité du roi » ${ }^{35}$. Le ministre ajoute : «le désir de faire des règlements, quoiqu'il parte souvent d'un bon principe, aveugle quelquefois au point de trouver mauvaises les dispositions les plus sages des loix du souverain, et de ne trouver bon que ce l'on fait ${ }^{36}$. Finalement (est-ce le souvenir de Macandal ? ${ }^{37}$ ), l'ordonnance sur les poisons fut appliquée malgré les représentations des conseillers ${ }^{38}$. Quelques années plus tard, alors que les administrateurs nouvellement nommés par le deuxième comité de législation sont favorables à l'atténuation du préjugé de couleur, les conseillers soutiennent leur action contre la population blanche du Mirebalais qui refuse de recevoir; dans une compagnie de dragons blancs, trois riches libres de couleur, les frères Montas. Point de vue de métropolitains ou de Créoles sensibles aux idées des Lumières? La récente thèse de Navarro-Andraud (2007) ne permet guère de percevoir les positionnements intellectuels et politiques des membres des conseils, elle confirme néanmoins la présence de Créoles, comme Moreau de Saint-Méry, parmi les conseillers, même si leur proportion reste à préciser ${ }^{39}$.

Dans l'exercice de leur fonction de juge ${ }^{40}$, les conseillers supérieurs, comme les magistrats de première instance de la partie ouest de Saint-Domingue, n'ont pas pratiqué au civil une « justice de race » (Pluchon 1982, p. 178), privilégiant systématiquement les Blancs sur les Noirs, même si cela n'exclut pas des tentatives d'intimidation à l'extérieur du tribunal et sans doute au niveau des commandants de quartier. Au conseil supérieur de Port-au-Prince, entre 1776 et 1789, les juges donnent raison aux libres de couleur dans près de $60 \%$ des cas de litige contre un Blanc. Ils appliquent les mêmes règles de droit écrit qu'en métropole : ils réclament les mêmes pièces (acte authentique ou acte privé reconnu comme preuve pleine et suflisante) pour prendre leurs décisions et usent des mêmes pratiques quand celles-ci font défaut. L'existence de procédures, où le serment d'un libre de couleur, même Noir non métissé, est reçu contre un Blanc avec succès nous semble la preuve évidente que les juges ne considèrent pas qu'il existe une infériorité de principe des Noirs par rapport aux Blancs.

Enfin, au quotidien, les jurislateurs ${ }^{41}$ n'ont pas appliqué strictement les règlements discriminatoires en matière d'identification des personnes. Dans les 
contrats de mariage du Cap-Français, la liberté des clients libres de couleur est justifiée par la «possession d'état » et non par le recours à des titres écrits (certificat baptistaire ou acte d'affranchissement) comme le prévoit l'article VI du règlement de $1773^{42}$. Il est alors souvent impossible de savoir si on a à faire à un affranchi ou un libre de naissance. Les surnoms tirés de l'idiome africain prévus pour les affranchis et les enfants illégitimes n'ont pas été attribués avec toute la rigueur nécessaire pour permettre la création d'une onomastique de couleur spécifique. En outre, la rétroactivité de la mesure a été difficile à mettre en œuvre, quelques affranchis et quelques enfants illégitimes ont changé de nom, beaucoup ont gardé l'ancien. Les procédures de protestation en usurpation d'identité restent très rares, alors que les exemples d'enfants illégitimes de couleur portant des noms de Blancs sont pléthores. Dans le cas des nouveaux affranchis, la résistance des familles blanches est évidente ${ }^{43}$.

\section{CATÉGORIES ET 《 SOUS-RACISME 》}

L'un des critères essentiels du succès des processus de "racisation 》 des sociétés réside dans la pratique, à l'intérieur de la population dominée, de ce que l'on appelle la " cascade de mépris » ou le " sous-racisme » (Debbasch 1967, p. 119), tant cette attitude suppose l'intériorisation par celle-ci du préjugé de la population dominante racisante. Selon Yvan Debbasch (1967), le préjugé de couleur impose que l'on ne se fréquente qu'entre gens de même nuance de couleur. Il affirme que chaque sous-groupe de la communauté libre de couleur « se veut et se sent étranger à ceux que l'éthique raciste lui indique comme étant situés à des niveaux inférieurs au sien » (ibid., p. 307). Qu'en est-il ?

Tout processus de sous-racisme nécessite d'identifier les autres individus en fonction d'une palette de couleurs ou catégories de couleur ou de métissage sur laquelle il convient de s'arrêter en préalable. Sur cette thématique, la classification de Moreau de Saint-Méry, publiée en 1797, occupe une place plus importante dans le débat qu'elle ne le devrait. On oublie trop souvent qu'elle est une production littéraire qui s'inscrit dans un projet de légitimation de l'esclavage et de subordination de la population libre de couleur. Elle ne reflète donc pas la réalité. De la fin du xvII ${ }^{\mathrm{e}}$ siècle au début de la Révolution, l'administration coloniale ne connaît que quatre catégories : les " nègres libres ", les " mulâtres libres », les «quarterons libres » et les « Blancs ». Les jurislateurs (notaires, greffiers), qui sont toujours plus proches des locaux, utilisent cependant une palette de couleurs plus large avec sept nuances ("nègre ", "mulâtre ", " grif » ${ }^{44}$, « quarteron », « tierceron » ${ }^{45}$, « mestif » ${ }^{46}$ et "sang-mêlé »), auxquelles il conviendrait d'en ajouter deux autres : "blanc», mais le terme n'est jamais indiqué dans les actes, et « indien » ou « sauvage » pour les rares Amérindiens. Ces catégories, plus variées que celles du législateur, prouvent l'importance 
accordée par les locaux à la couleur des individus. Le nuancier est en effet spécifique, il se distingue de celui de la Martinique, de la Guadeloupe et de la Guyane, lesquels sont d'ailleurs tous différents entre eux. Certains termes sont inspirés de l'espagnol ${ }^{47}$, mais pas toujours avec les mêmes significations. Cette palette a aussi évolué dans le temps. En 1765, dans le cadre de la réforme des milices, le gouverneur d'Estaing propose d'intégrer les enfants des mestifs dans les compagnies des Blancs, « car les mestifs forment la dernière classe des gens de couleur » (Moreau De Saint-Méry 1784-1790, tome IV, article 28 de l'ordonnance générale des milices du 15 janvier 1765). En 1777, Hilliard d'Auberteuil $(1777$, p. 82) tentant d'évoquer des individus au sixième rang de métissage parle de la « petite-fille d'une mestive », là où, en 1797, Moreau de Saint-Méry utiliserait le terme « quarteronnée ». Tenté à son tour d'évoquer un stade plus lointain, Moreau de Saint-Méry, en 1797, fournit une mauvaise périphrase «sang-mêlé qui s'approche continuellement du blanc » (Moreau de Saint-Méry 1984, tome 1, p. 86). À l'évidence, à ce stade et d'ailleurs bien avant, les passages de la ligne (l'intégration des libres de couleur dans la catégorie des "Blancs 》 ou leur assimiliation aux Blancs) sont sans doute extrêmement fréquents, même si certains dont l'origine paraît suspecte ne sont pas à l'abri de procès ou de vexations ${ }^{48}$. D'une manière générale, Hilliard d'Auberteuil, Alexandre-Stanislas de Wimpffen, Moreau de Saint-Méry, Julien Raimond ne sont pas tous d'accord sur l'aspect des individus qui sont qualifiés de «sang mêlé », de "mestif » ou de « tierceron ». Cette dernière catégorie n'est même pas citée dans la classification de Moreau de Saint-Méry, alors que nous avons trouvé dans les archives notariales et le greffe plus d'une trentaine d'individus qualifiés ainsi (Rogers 1999, chap. 5).

L'existence d'une palette de couleurs extrêmement variée induit-elle des relations hiérarchisées en fonction de la couleur des individus ? Ainsi que nous l'avions évoqué dans un précédent article (Rogers 2003), au quotidien, les hommes de couleur et les autres sont souvent beaucoup plus pragmatiques, et cela aussi bien chez les plus modestes que chez d'autres plus aisés.

Pour ce qui est des esclaves, notre connaissance est tout à fait embryonnaire. Ordinairement, les historiens ${ }^{49}$ affiriment l'existence d'une hiérarchie interne au monde servile où les métis sont toujours favorisés par rapport aux Noirs non-métissés. Pour Saint-Domingue, ils citent souvent Moreau de Saint-Méry dans la Description... française de l'isle de Saint-Domingue ou dans son mémoire anonyme d'un planteur de Saint-Domingue. Dans les deux cas, le juriste créole affirme :

Il n'est pas un nègre qui osa acheter un mulâtre ou un quarteron pour s'en faire servir. Si cette tentation avait lieu, le quarteron esclave préfèrerait le parti le plus violent, la mort même, à un état qui le déshonorerait de sa propre opinion, et tous ceux de sa caste se croiraient obligés de seconder ses projets, parce qu'ils partageraient son infamie. 
L'anecdote est saisissante, mais elle n'exprime que le préjugé de celui qui la relate. La réalité domingoise le contredit maintes et maintes fois. Même si les métis sont assez peu nombreux dans la population servile, les cas de Noirs libres achetant des métis enfants ou adultes sont bien réels, ceux d'esclaves métis ayant survécu chez leurs propriétaires plus foncés aussi. Notons l'exemple, en 1783, de la négresse libre Marie-Jeanne Niady acquérant, pour 2700 livres, Suzanne, mulâtresse créole de 28 ou 30 ans, et sa fille de trois ans, Nanette ${ }^{50}$. De même, en 1788, Pauline, négresse libre, achète, pour 5280 livres, un mulâtre, maître d'hôtel et confiseur du célèbre Barré de Saint-Vénant, membre de la chambre d'agriculture du Cap ${ }^{51}$. En 1777, lorsque le grif libre Simon Mansel décide d'épouser son esclave, plus claire que lui, Rosalie, mulâtresse, leurs amis, les nègre libres Jean-Baptiste et Barbe Mouton, " pour marquer leur contentement " ${ }^{52}$, font don au jeune couple de deux esclaves de sexe féminin, de 582 livres de meubles et effets divers et d'une somme de 1782 livres qui restera la propriété personnelle de la jeune fiancée, en plus du douaire promis par le futur conjoint. Où est l'ostracisme, le sentiment d'infamie ? Enfin, il conviendrait aussi de se rappeler qu'à la différence de ce qui se passe dans les petites Antilles, les planteurs blancs de SaintDomingue pendant les deux derniers tiers du XvIII ${ }^{c}$ siècle confient encore des postes de commandeur à des Noirs non métissés et même, plus souvent, à des Africains qu'à des Créoles (Debien 1974, p. 122) ${ }^{53}$. De même, nombre d'ouvriers de talent, de chefs d'équipes ou de domestiques attachés à la personne des maîtres, en ville comme à la campagne, sont des Africains ou des nègres créoles et non des métis. Tous ces éléments ne favorisent pas le développement systématique d'une logique de discrimination favorable aux plus clairs par rapport aux plus foncés, sur la base de capacités intellectuelles soi-disant inférieures ou supérieures.

Du côté des libres de couleur, les faits le confirment aussi. Parmi les partenaires économiques ${ }^{54}$ de Julien Raimond, John Garrigus (1996, p. 17) a signalé l'existence de Blancs, de mulâtres, mais aussi d'une négresse libre, originaire du Curaçao. Anne-Dominique Acquiez, qui a été affranchie à l'âge de 25 ans, possède à Aquin une auberge et une boutique en 1770. Pendant trois ans et demi, Julien Raimond a pris ses repas dans son établissement et, en 1775, s'est fait livrer quelque 402 livres de marchandises. Entre eux, des liens plus personnels se sont tissés. En 1773, Julien sert de témoin pour authentifier son testament. Plus important, son frère, Guillaume Raimond, signe son contrat de mariage, en grande pompe, chez « Mama Acquiez ». Au-delà des différences pigmentaires, la familiarité semble possible.

Dans les villes de Saint-Domingue, la ségrégation résidentielle n'existe pas. Au quartier de la Petite Guinée du Cap, seuls $46 \%$ des bailleurs ou des locataires sont des nègres libres, les $54 \%$ restant se répartissent presque également entre Blancs et métis. Comme à Port-au-Prince, les Blancs, les Noirs et les métis sont présents dans tous les quartiers. Du fait de la configuration particulière des 
maisons domingoises, propriétaires et locataires partagent souvent la cour, la cuisine et le puits, en toute simplicité. D'autres vivent effectivement sous le même toit, sans qu'il y ait concubinage. Entre juin et juillet 1784, le quarteron libre Joseph Pironneau sous-loue plusieurs appartements d'une maison donnant sur la rue Royale et sur la rue des Religieuses : le sieur Constantin Parfait prend une chambre et deux cabinets sur la rue Royale ; le sieur Lasserre, une seule chambre sur la même rue ; les négresses libres Marie-Louise A. Traitté et Marie-Louise Tardivy choisissent chacune une chambre et un cabinet sur la rue des Religieuses. Le montage est prévu pour le long terme : seul le bail de Marie-Louise A. Traitté n'est que de trois ans, les autres ont une durée de sept ans ${ }^{55}$. Si, à Port-au-Prince, les propriétaires métis privilégient souvent les locataires blancs, le quarteron Joseph Pironneau semble ici faire pleinement confiance à des négresses libres.

Dans les villes comme dans les campagnes, la proximité résidentielle a permis le développement de relations de voisinage, de confiance et d'amitié parfois qui suggèrent le nivellement des différences d'autant plus qu'elles ne s'inscrivent pas dans des rapports hiérarchiques forts. Au Cap-Français, le choix du maître d'apprentissage se fait indépendamment de la couleur : depuis le petit Blanc ${ }^{56}$ confié à un quarteron jusqu'au petit mestif confié à un nègre libre. Au moment de choisir la personne qui rachètera et/ou éduquera leurs enfants encore esclaves, les négresses libres du Cap-Français font confiance, dans $40 \%$ des cas, à un tuteur blanc ou métis, souvent d'ailleurs sans compensation financière. Ainsi, "en reconnaissance de la bonne amitié qu'elle lui porte et des services qu'elle lui a rendus depuis cinq ans qu'elle la loge, la nourrit, et qu'elle continue à lui rendre journellement » (Rogers 1999, p. 582), Marie-Jeanne, négresse libre du Cap, lègue tous ses biens à la dame Veuve Cottin, « mulâtresse libre, sa sincère amie ». Plus largement, les testaments mentionnent aussi des petits prêts du quotidien consentis sans reconnaissance de dette, ainsi que des legs accordés à des amis ou à des filleuls d'une couleur différente. En 1784, dans la paroisse isolée du Baynet, le nègre libre François Tourat ${ }^{57}$, sellier de profession, favorise ainsi un petit mulâtre Jean-François Fillières, une petite négrille, Marie-Françoise Fillières, sa demisœur, et une quarteronne Marie-Henriette Tourat.

Selon Bonniol et Benoist (Bonniol 1992, 2001 ; Bonniol et Benoist 1994), l'une des caractéristiques les plus fondamentales des sociétés marquées par un ordre socioracial s'apprécie au niveau des pratiques matrimoniales ou reproductives. La sélection du conjoint s'établit «en fonction de l'origine et/ou des caractères physiques » des individus et participe donc à la reproduction de l'ordre socioracial imposé. À cet égard, dans l'état actuel de la recherche, SaintDomingue se singularise une fois de plus par rapport aux autres colonies françaises. Les mariages mixtes, jamais interdits, s'y pratiquent pendant toute la période. En 1777, Hilliard d'Auberteuil (1777, p. 79) affirme qu'il y a « environ trois cent [sic] blancs mariés à des filles de sang-mêlé, [et que] plusieurs sont nés gentilshommes ». En 1780, Pierre-Ulrich Dubuisson (1780, p. 59) renchérit : 
L'indigence confond de toute part les rangs et les couleurs : la fille unique d'un nègre ou d'un mulâtre propriétaire du plus petit terrein [sic] sera recherchée par vingt de ces misérables cadets, nés sur des terres qui, après avoir été leur berceau, ne seront jamais leur demeure.

En fait, les mariages mixtes restent assez fréquents à la fin de l'Ancien Régime : ils représentent $17 \%$ des unions légitimes dans le Sud (Houdaille 1963), $7 \%$ des contrats de mariages à Port-au-Prince et $11 \%$ au Cap-Français ${ }^{58}$. En outre, ils s'observent dans des milieux divers, modestes mais aussi plus aisés. En matière de concubinage, le faible nombre de femmes blanches favorise les femmes de couleur, qui sont le plus souvent l'unique compagne de leur partenaire blanc. La jurisprudence des conseils supérieurs de Saint-Domingue très favorable aux enfants naturels simples assure à ces enfants des revenus parfois très importants et suggère que le concubinage est un état aménagé, moins ostracisant qu'on ne le suppose ordinairement.

Les contrats de mariage entre personnes de couleur que nous avons étudiés confirment l'importance de l'identité pigmentaire, davantage entre les Noirs non-métissés et les métis, qu'entre individus relevant de différentes catégories de métissage. De manière symptomatique, les grifs sont très peu nombreux dans la population libre de couleur. Ils représentent moins de $2 \%$ de la clientèle des notaires du Cap-Français contre $56,5 \%$ pour les nègres libres et moins de $4 \%$ de la clientèle de couleur de Port-au-Prince, contre 39,2\% pour les nègres libres et $44,3 \%$ pour les mulâtres. D'une manière générale, on épouse quelqu'un de sa nuance de couleur dans près de $80 \%$ des cas au Cap-Français et $70 \%$ à Port-auPrince. Si l'on ne prend en compte que les nègres libres, ce taux s'élève respectivement à $90 \%$ et $86 \%$. En revanche, les métis du Cap-Français ne respectent la norme que dans $61 \%$ des cas, contre $54,5 \%$ à Port-au-Prince. À l'évidence, les transgressions sont aisées entre métis. Le quarteron Julien Raimond et sa deuxième femme, la mulâtresse Françoise Dasmard-Challe, en sont d'ailleurs un bon exemple. Souvent, les transgressions concernent les milieux assez fortunés. En 1785, la négresse libre Anne Barthélémy dite Sancié ${ }^{59}$ apporte une dot de près de 40000 livres (quatre esclaves offertes par sa mère et 32000 livres de biens propres en esclaves, argent et divers effets personnels, dont des bijoux). Comme dans les riches mariages mixtes, des précautions sont prises et la communauté est limitée à 12000 livres. Cette étude a été menée uniquement à partir des contrats de mariage, il conviendrait sans doute de l'élargir avant de conclure.

Peut-on déduire de cette situation l'existence d'un «sous-racisme » (Debbasch 1967, p. 118) ou l'intériorisation du préjugé de couleur ? Si le racisme suppose l'existence de groupes d'individus supérieurs à d'autres et légitime la domination des premiers sur les seconds, il nous semble qu'il faut répondre par la négative, du moins entre gens de couleur. La proximité des relations des uns et des autres au quotidien l'interdit. S'il implique peur et mépris de l'autre, il nous faut aussi répondre négativement. De manière exemplaire, lors de la signature de $25 \%$ 
des contrats de mariage au Cap, l'assistance est composée de personnes de toutes les couleurs. Certaines sont des voisins de la Petite Guinée, du Haut-du-Cap pour Geneviève Scipion, d'autres des artisans d'un même secteur professionnel. En 1788, le mariage de Marie-Noëlle, négresse libre, et de Louis Rodin, mulâtre libre, scelle une union déjà féconde. La marraine et le parrain du petit garçon sont là : l'un est quarteron, l'autre mulâtresse. Un négociant blanc est parmi les témoins ainsi que deux mulâtres. La mère de Marie-Noëlle, Marie-Catherine dite Angélique surnommée Fabia, une négresse, affranchie en 1783, est aussi présente. On mentionne enfin une autre négresse libre, la sœur utérine de l'époux. À l'évidence, les nuances n'ont pas l'air de gêner les uns et les autres. Dans la petite paroisse rurale de Baynet, partie sud de la colonie, en $1783^{60}$, la négresse MarieMagdeleine Denis ouvre sa maison au quarteron Antoine Lavoile et à la demoiselle Marie-Jeanne Moutard, mulâtresse libre, pour la signature de leur contrat de mariage. Dans la partie ouest, la même année, le mulâtre libre Jean Pouillot, surnommé Azor, connu sous le nom de Bernon ${ }^{61}$, au moment de rédiger son testament n'oublie aucun de ses parents en esclavage, quelle que soit leur couleur : sa mère Guitonne, négresse créole, âgée de 72 ans, mais aussi son jeune "frère naturel » Hyppolite, nègre créole, âgé de trente ans. Tous sont confiés à la garde d'un quarteron libre Pierre Gabriel Pellerin, cordonnier de Croix-des-Bouquets, chargé d'obtenir leur liberté. Cette situation ne prévaut pas dans toute l'île et notre propos ne saurait s'appliquer à son ensemble même s'il n'est pas seulement valable pour le monde urbain. Il y a sans doute une variété de situations, liées à des contextes sociaux distincts, que seules des études complémentaires permettront de préciser. Dans les campagnes du Nord (King 2000, p. 223) ou de l'Ouest notamment, il semble que les grandes familles métisses les plus riches tendent à se structurer de manière plus classique en s'alliant à d'autres familles de leur couleur, plus même qu'à des Blancs.

\section{Conclusion}

Les hommes de la fin du $\mathrm{XVII}^{\mathrm{e}}$ siècle qui conçoivent et soutiennent le projet administratif de réorganisation de la société domingoise dans un sens discriminatoire n'ont pas les connaissances biologiques ou génétiques pour élaborer une théorie discriminatoire de type racial, comme ont pu le faire les théoriciens des $\mathrm{XIX}^{\mathrm{e}}$ et $\mathrm{Xx}^{\mathrm{e}}$ siècles, même si certains s'en approchent (Praslin, Bourgeois de Boynes, Moreau de Saint-Méry par quelques aspects). Leur projet participe sans doute par certains côtés davantage d'une tentative de réaction face à une société locale dont le fonctionnement quotidien apparaît comme potentiellement dangereux pour le maintien de l'ordre esclavagiste, en ce qu'il est constamment en contradiction avec un ordre socioracial binaire. Dès lors, projeter une grille explicative racialiste simple pour rendre compte de la complexité, de la «schi- 
zophrénie » ${ }^{62}$ - voire simplement de la pluralité d'opinions d'une société où certes des discriminations que nous appelons raciales existent de manière patente, mais où d'autres types de relations sont aussi possibles de manière ordinaire entre gens de couleur différente - nous semble tout à fait insuffisant. À partir du moment où les stratégies de contournement et, plus largement, les comportements ordinaires n'obéissent plus, sur une large échelle, à la logique administrative discriminatoire, la question fondamentale des critères pertinents pour qualifier une société de « racisante » se pose à nouveau. Les travaux de Barbara Fields (1990) sur les questions raciales aux États-Unis d'Amérique offrent peut-être une hypothèse suggestive pour appréhender la situation domingoise. À la fin du $\mathrm{XVIII}^{\mathrm{e}}$ siècle, nous serions ainsi à ce moment charnière où l'idéologie de la race se met en place et où la société locale hésite encore sur le fonctionnement social à adopter. À ce stade intermédiaire, toutes ses composantes n'auraient pas encore intégré cette " routine comportementale ${ }^{63}$ qui permet de vérifier au quotidien l'application de cette idéologie, ce qui expliquerait la pluralité des discours et les comportements peu conformes.

Nous ne prétendons pas avoir donné de réponse définitive. Cependant, la difficulté de certains à accepter la complexité de la société domingoise que nous avons tenté de décrire s'ancre sans doute dans un traitement téléologique de l'histoire, où l'on voudrait trouver dans la société locale de la fin de l'Ancien Régime toutes les explications de la période révolutionnaire. Dans le cas domingois, néanmoins, cette démarche, a priori acceptable, est biaisée par une perception racialisée très forte, mais très réductrice de la révolution haïtienne. Les carrières bien différentes d'un Jean Kina (Geggus 2002), d'un Jean-François, leader de l'insurrection de 1791 puis généralissime des troupes espagnoles, ou d'un Toussaint Louverture engagent en effet $a$ minima à la prudence. La présence de Blancs comme Ferrand de Baudière ou Jean-Baptiste Gérard ${ }^{64}$ aux côtés des libres de couleur dès 1789 ou aux cotés des anciens esclaves pour les troupes polonaises du général Leclerc suggère aussi la complexité d'un très long conflit où la couleur est loin d'être le seul critère explicatif des événements. Dès lors, ces quelques pages invitent à approfondir l'analyse des différents groupes sociaux domingois, notamment la population blanche, au-delà des seules élites, mais aussi celle des esclaves, en tenant compte de la diversité géographique et organisationnelle de la société locale. Mieux cerner les ressorts des politiques administratives avant, pendant et après la mise en place du projet des années 1760-1770 apparaît également essentiel. Enfin, s'il y a racisation à partir des années 1760 , quelle est la nature de la situation antérieure ? Quels rapports y a-t-il entre le fonctionnement social avant 1789 ou 1791 et la variété des réactions et des évolutions pendant la Révolution et après ?*

\footnotetext{
* Manuscrit reçu en juin 2008, accepté pour publication en juin 2009.
} 


\section{Notes}

1. Il s'agit d'une personne noire ou métisse libre de naissance ou par affranchissement.

2. Par des auteurs allant de Thomas Madiou à Doris Garaway en passant par Cyril L. R. James, Yvan Debbasch, David Nicholls, Jean-Luc Bonniol. À titre d'exemples récents, on pourra se reporter aux travaux de Garaway (2005, p. I) et de Bonniol (1992, p. 77).

3. Le Code noir de 1724 y était beaucoup plus radical que celui de 1685 qui avait cours dans les Antilles françaises.

4. Une déclaration du 8 février 1726 étend aux îles du Vent (Martinique et Guadeloupe) les dispositions de l'article 52 du Code noir de 1724, lequel article interdit les donations entre " vifs » (personnes vivantes) au profit des hommes de couleur. Une déclaration du 3 octobre 1730 impose aux hommes de couleur le paiement d'une capitation. L'article 6 de l'ordonnance des administrateurs de la Martinique réactive la disposition en 1766 (Petit 1777, pp. 266, 253).

5. À partir de la fin du XvıI ${ }^{\mathrm{e}}$ siècle, de nombreuses mesures sont prises pour limiter le séjour des esclaves, puis plus généralement des Noirs et gens de couleur dans la métropole : édit du 25 octobre 1716, déclaration royale du 15 décembre 1738 , ordonnances des 31 mars, 5 avril et 30 juin $1763 \ldots$

6. L'interdiction date de 1733, mais elle n'a vraiment été appliquée qu'à partir de 1767.

7. Sur l'application de ce règlement, voir Rogers (1999, chap. 5).

8. Archives nationales d'Outre-Mer (ANOM), fonds Colonies, Collection Moreau de Saint-Méry, F3 72, folio 46 .

9. Archives nationales, fonds Colonies, B 141, folio 193, $\mathrm{v}^{\circ}-195,26$ novembre 1772, lettre de Boynes à Vallières et Montarcher (cité in Tarrade 1991, p. 380).

10. Archives nationales, fonds Colonies, Collection Moreau de Saint-Méry, F3/72, notamment folio 46 .

11. Archives nationales d'Outre-Mer (ANOM), fonds Colonies, Collection Moreau de SaintMéry, F3/72, folio 241. Instructions au gouverneur du Chilleau du 1/8/1788.

12. La définition du terme est évidemment plus large, comme le rappelle l'article " race " du dictionnaire Le Robert, édition 2004 : « groupe ethnique qui se diffërencie des autres par un ensemble de caractères physiques héréditaires (couleur de la peau, forme de la tête, proportion des groupes sanguins) représentant des variations au sein d'une même espèce ». En général, elle désigne les individus selon trois catégories : " Blancs », « Jaunes » et " Noirs ».

13. Rousselot de Surgy et le vicomte de Mirabeau sont cités par Pierre Boulle (1988).

14. Archives nationales, F3/139, folio 289, réflexions sur les moyens de rendre meilleur l'état des nègres et des affranchis des colonies, mémoire dit de Saint-Lambert.

15. «C'est la croyance en la différenciation bio-physique et non l'apparence qui est impliquée [dans le racisme] » (Guillaumin 2002 p. 95). «La biologisation de la perception, dès qu'elle est associée à la perception de la différence sociale, forme le nœud de l'organisation raciste » (ibid., p. 96).

16. Cité par Pierre Pluchon (in Wimpffen 1993, p. 34).

17. John Garrigus (2006, p. 113), qui a étudié l'ensemble des écrits du Domingois Émilien Petit, démontre de manière assez convaincante que "Petit's racism was not primarily biological, but driven by" the need to orient colonial "patriotism" toward France » [ " le racisme de Petit n'est pas à l'origine d'ordre biologique, mais lié au besoin d'orienter le patriotisme colonial vers la France » (traduction de l'auteur, D. R.)].

18. L'exemption de la capitation obtenue par les libres de Gorée faisait coexister deux types de libres de couleur à la Guyane. Ils durent finalement payer la capitation, mais ils purent aller en métropole sans demander l'autorisation des administrateurs (Debbasch 1967, pp. 55-56).

19. Archives nationales, fonds Colonies, Collection Moreau de Saint-Méry, F3/91, instructions aux administrateurs, messieurs Fieldmont et Malouet en 1776, p. 209.

20. La formulation exacte de Federica Morelli (communication personnelle 2006) est la suivante : " tentative, typique des sociétés d'Ancien Régime, de mettre de l'ordre dans une situation confuse au 
sein d'une société corporatiste et hiérarchisée, [plus] que [d]'une véritable tentative de "racialisation" de la société dans le sens de créer des catégories visant à engendrer des logiques ségrégationnistes ".

21. Voir la lettre de Reynaud de Villeverd du 21 février 1781 aux commandants en second (citée in Pluchon 1991, p. 407).

22. Voir la lettre co-signée par l'intendant de Montarcher au sénéchal du Cap en date du 9 juillet 1772 (citée in Pluchon 1991, p. 407).

23. Voir la lettre de Reynaud de Villeverd du 21 février 1781 aux commandants en second de la colonie (citée in Pluchon 1991, p. 407).

24. Sur ce projet, voir Garrigus (2006, pp. 109-116).

25. On pourra se reporter à la communication intitulée «Races, ethnies et communautés : la Guyane et Saint-Domingue en miroir » que Marie-José Jolivet a prononcée pour le colloque «Des catégories et de leurs usages dans la construction sociale d'un groupe de référence : "race", "ethnie" et "communauté" aux Amériques », décembre 2006, EHESS, Paris.

26. Sur les variations des politiques françaises d'assimilation des populations amérindiennes, voir Belmessous (2004) et Aubert (2004).

27. Voir Petit, Traité sur le gouvernement des esclaves, lettre du ministre du 7 janvier 1767, p. 258.

28. "Les conséquences du préjugé [nous semblent] plus importantes que leur justification idéologique » (traduction de l'auteur, D. R.).

29. Voir Peabody (1996) et Noël (2006).

30. Voir Boulle (1988) et Elisabeth (1954).

31. L'article 28 de l'ordonnance générale des milices du 15 janvier 1765 propose que les « enfants des mestifs servent dans la milice avec les Blancs " (voir Moreau de Saint-Méry 1784-1790, tome IV). Pour une étude détaillée, voir Frostin (1973, pp. 669 et sq.).

32. Moreau de Saint-Méry 1784-1790, tome V, article V de l'Ordonnance sur les poisons.

33. Archives nationales, fonds Colonies, C9a 151, le 4/5/1781.

34. Les notaires mentionnent plusieurs clients de couleur exerçant cette profession jusqu’à la fin de la période.

35. Archives nationales d'Outre-Mer (ANOM), fonds Colonies, F3/78, p. 249.

36. Archives nationales d'Outre-Mer (ANOM), fonds Colonies, F3/78, p. 249.

37. Macandal était un esclave de la partie Nord à qui on avait attribué des vagues d'empoisonnement et dont la traque avait duré plusieurs années en raison de nombreuses complicités dans la population servile. Le souvenir de son épopée était encore vivante en 1791 chez les esclaves comme chez les maîtres.

38. Mars 1781 : refus d'enregistrer l'ordonnance sur les poisons, après la sommation du gouverneur, envoi de représentations signées par Bourdon et Volunbrun, le 28 avril. Archives nationales, fonds Colonies, C9a/151, le 28/4/1781. Mai 1781 : idem pour l'ordonnance sur les orfèvres.

39. Elle note la présence d'au moins 12 Créoles parmi les 95 conseillers, présidents, assesseurs, et procureurs des deux conseils de Saint-Domingue qu'elle a identifiés pour la période de 1763 à 1792 . Les autres sont, pour 26, des métropolitains et l'origine des 51 autres est inconnue (voir Navarro-Andraud 2007).

40. Sur les libres et la justice du roi, voir Rogers (1999, chap. 5).

41. Le terme de « jurislateur » se ditingue du terme « législateur » en ce que le second désigne les instances qui font la loi, alors que le premier évoque celles qui ne font que la mettre en pratique (ici greffiers et notaires).

42. Voir Moreau de Saint-Méry 1784-1790, tome IV, article 6 du règlement de 1773 sur l'identification des personnes.

43. Sur l'application du règlement de 1773 à Saint-Domingue, voir Rogers (1999, chap. 5).

44. Dans l'usage ordinaire, le terme " grif » désigne un individu de sexe masculin dont l'un des parents est noir non métissé et l'autre mulâtre.

45. À Saint-Domingue, au xvı" siècle, le terme " mestif » désigne, en règle générale, un individu né d'un parent blanc et l'autre quarteron, alors que le mot « tierceron " évoque une personne dont l'un des 
parents est quarteron et l'autre mulâtre. Le "sang-mêlé ", cas très rare, correspond, dans le schéma le plus simple, à un individu dont l'un des parents est mestif et l'autre blanc. Sur la palette des couleurs domingoises, voir Rogers (1999, chap. 5).

46. Pour éviter les confusions, dans cet article, le terme "mestif " renvoie à une catégorie de désignation domingoise précise et non à un groupe générique incluant tous les sangs-mêlés quelle que soit leur nuance de couleur.

47. Les termes " mulâtre », " grif » et " quarteron » sont calqués sur l'espagnol, avec respectivement mulato, grifo, cuarterón (Lavallé 1993, p. 153).

48. Sur la catégorie des suspects, voir Debbasch (1967, pp. 66-67) et Ogle (2006, pp. 23-46).

49. Pour un point de vue similaire récent, voir Régent (2004).

50. Archives nationales d'Outre-Mer (ANOM), fonds Colonies, notsdom 200, vente du 18/2/1788.

51. Archives nationales d'Outre-Mer (ANOM), fonds Colonies, notsdom 200, vente du 18/2/1788.

52. Archives nationales d'Outre-Mer (ANOM), fonds Colonies, notariat de Saint-Domingue, Grimperel ancienne cote 845, vente du 18/2/1788.

53. Pendant la Révolution, Jacques Houdaille (1963) a observé une légère préférence pour les Créoles sur les Africains. Plus de $50 \%$ des 150 ateliers analysés étaient dirigés par des Créoles dans la zone occupée par les Anglais. Geggus (1999, p. 41) suggère qu'à la veille de la Révolution les commandeurs de la partie nord étaient principalement des Créoles dans les exploitations de plaine plus anciennes et donc très créolisées ( 3 Créoles sur 5), mais plutôt des Africains dans les exploitations des mornes, souvent beaucoup plus récentes et pourvues d'ateliers composés de plus d'Africains.

54. Voir l'inventaire de ses 159 papiers commerciaux déposés, en 1786, chez le notaire Paillou.

55. Archives nationales d'Outre-Mer, DPPC, notariat de Saint-Domingue, Rivery registre 1545, testament du 12 décembre 1777.

56. Un cas unique. Archives nationales d'Outre-Mer (ANOM), fonds Colonies, 176, Bordier jeune, contrat d'apprentissage du 12/10/1779.

57. Archives nationales d'Outre-Mer (ANOM), fonds Colonies, 649 Funuel de Séranon, acte $\mathrm{n}^{\circ} 119$, testament du 15/12/1784.

58. Soit 7 cas sur 68 à Port-au-Prince et 12 pour 110 au Cap-Français.

59. Archives nationales d'Outre-Mer (ANOM), fonds Colonies, notsdom 1521, Porée, contrat du $18 / 10 / 1785$.

60. Archives nationales d'Outre-Mer (ANOM), fonds Colonies, ancienne cote, Funuel de Séranon, 645 , acte $\mathrm{n}^{\circ} 26$ du $25 / 9 / 1783$

61. Archives nationales d'Outre-Mer (ANOM), fonds Colonies, Guieu, notsdom 889, acte n ${ }^{\circ} 562$, testament du 28 août 1783.

62. Pérotin-Dumon (1998, p. 643) évoque une «schizophrénie vécue intimement, mais aussi collectivement entre interdits officiels et liens de fait ").

63. Sur cette idée, voir Fields (1990, p. 114).

64. Sur Jean-Baptiste Gérard, se reporter à l'ouvrage de Jacques de Cauna (1998, pp. 378-381).

\section{RÉFÉRENCES CITÉES}

Abbé RaYNAL

$1781 \quad$ Histoire philosophique et politique des établissements et du commerce des Européens dans les deux Indes, tome 2, Jean Léonard Pellet, Genève.

ANDERSON Jeffrey Elton

2005 "Ethnicity, nationality, and race in colonial America », in Alton Hornby Jr (éd.), A companion to African American history, Blackwell publishing, Oxford, UK. 
Aubert Guillaume

2004 " The blood of France : race and purity of blood in the French Atlantic world ", The William and Mary Quaterly, 61 (3), pp. 439-478.

BeLmessous Saliha

2004 «Être Français en Nouvelle-France : identité française et identité coloniale aux dix-septième et dix-huitième siècles ", French Historical Studies, 27 (3), pp. 507-540.

BonNiol Jean-Luc

2007 "Racialisation ? Le cas de la colorisation coloniale des rapports sociaux ", Faire savoirs, 6, pp. 37-46.

BonNiol Jean-Luc (éd.)

1992 La couleur comme maléfice, une illustration créole de la généalogie des Blancs et des Noirs, Albin Michel, Paris.

2001 Paradoxes du métissage, CTHS, Paris.

Bonniol Jean-Luc et Jean BeNoIST

1994 «Hérédités plurielles. Représentations populaires et conceptions savantes du métissage », Ethnologie française, XXIV (1), pp. 58-69.

Boulle Pierre

1988 "La naissance du racisme en Europe: quelques hypothèses ", in Serge Daget (éd.), De la traite à l'esclavage du $v^{f}$ au $x x^{e}$ siècle. Colloque international sur la traite des Noirs, 1985, Nantes, Centre de recherches sur le monde Atlantique/SFHO, Paris/Nantes, pp. 539-540.

1989 «Les gens de couleur à Paris à la veille de la Révolution », in Michel Vovelle (éd.), Images de la Révolution française, Publications de la Sorbonne, Paris, tome 1, pp. 159-168.

2007 Race et esclavage dans la France de l'Ancien Régime, Perrin, Paris.

Cauna Jacques de

1998 L'Eldorado des Aquitains, Gascons, Basques et Béarnais aux îles d'Amérique, $x$ xvI ${ }^{e}$-xvit $I^{e}$ siècles, Atlantica, Biarritz.

D'AuBERteuil Hilliard

1777 Considérations sur l'état présent de la colonie française de Saint-Domingue, Grangé, Paris.

DebBasch Yvan

1967 Couleur et liberté. Le jeu du critère ethnique dans un ordre juridique esclavagiste. Tome 1: L'affranchi dans les possessions françaises de la Caraibe, 1635-1833, Dalloz, Paris.

\section{Debien Gabriel}

1974 Les esclaves aux Antilles françaises, Société d'Histoire de la Guadeloupe, Basse Terre.

Debien Gabriel et Françoise ThÉsée

1975 Un colon niortais à Saint-Domingue : Jean Barré de Saint-Vénant (17371810), Imbert Nicolas, Niort. 


\section{Douglas Cope Robert}

1994 The limits of racial domination. Plebeian society in colonial Mexico city, 1660-1720, University of Wisconsin Press, Madison, Wisconsin.

Dubuisson Pierre-Ulrich

$1780 \quad$ Nouvelles considérations sur Saint-Domingue en réponse à monsieur Hilliard d'Auberteuil, CELLOT, Paris.

\section{ELISABETH Leo}

1954 Les problèmes des gens de couleur de Saint-Domingue à Bordeaux sous l'Ancien Régime (1716-1787), DES, Bordeaux.

FIELDS Barbara J.

1990 "Slavery, race and ideology in the United States of America ", New Left Review, 181, pp. 95-118.

\section{Frostin Charles}

1973 Histoire de l'autonomisme colon de la partie française de Saint-Domingue aux xvif et $x v$ IIt siècles: contribution à l'étude du sentiment américain d'indépendance, thèse de doctorat, Université de Paris 1.

\section{GARAWAY Doris Lorraine}

2005 The libertine colony : creolization in the early French Caribbean, Duke University Press, Durham.

\section{GARRIGUS John}

1996 "Color and class on the eve of the Haitian revolution: SaintDomingue's free colored elite as colons Américains ", Slavery and Abolition, 17, pp. 20-43.

2006 Before Haiti : race and citizenship in French Saint-Domingue, Palgrave MacMillan, New York.

\section{GegGus David}

1999 "Slave societies in the sugar plantation zones of Saint-Domingue and the Revolution of 1791-1793 », Slavery and Abolition, 20, august, pp. 31-46.

2002 "Slave, soldier and rebel. The strange career of Jean Kina », in David Geggus (éd.), Haitian revolutionary studies, Indiana University Press, Bloomington, pp. 137-151.

\section{Guillaumin Colette}

2002 L'idéologie raciste, Gallimard, coll. « folio essais », Paris.

HoudAiLle Jacques

1963 "Trois paroisses de Saint-Domingue au $\mathrm{xVIII}^{\mathrm{e}}$ siècle », Population, 18, pp. 93-110.

JACQUART Albert

1974 La génétique des populations humaines, PUF, Paris.

Jounnna Arlette

2003 "Race », in Lucien Bély (éd.), Dictionnaire de l'Ancien Régime, PUF, Paris. 
KING Stewart

2000 Blue coat or powdered wig: free people of color in pre-revolutionary SaintDomingue, University of Georgia Press, Athens, GA.

LAVALLé Bernard

1993 L'Amérique espagnole de Colomb à Bolivar, Belin Histoire Supérieur, Paris.

LEwONTIN Richard C.

1974 The Genetic Basis of Evolutionary Change, Columbia University Press, New York.

Leivontin Richard C., Steven Rose, Peter Russel and Léon Kamin

$1984 \quad$ Not in our genes, Penguin, Harmondsworth UK.

Malouet Pierre-Victor

1803 "Essai sur l'administration de Saint-Domingue », in Abbé Raynal, Collections de mémoires sur les colonies et en particulier sur Saint-Domingue, tome 4, Baudouin, Paris [1785].

MAurel Blanche (éd.)

1935 Cahiers de doléances de la colonie de Saint-Domingue, imprimerie Mellotée, Châteauroux/Ernest Leroux, Paris.

\section{Moreau de SAINT-Méry Médéric-Louis-Elie}

1784-1790 Loix et constitutions des colonies françaises de l'Amérique sous le vent, [s. n.], Paris.

1984 Description topographique, physique, civile, politique et historique de la partie française de l'isle de Saint-Domingue, réédité par Blanche Maurel et Étienne Taillemite, Société française d'histoire d'Outre-Mer, Paris.

2004 Description topographique, physique, civile, politique et historique de la partie française de l'isle de Saint-Domingue, nouvelle édition présentée par Marcel Dorigny, Étienne Taillemite et Philippe Hrodej, Société française d'histoire d'Outre-Mer, Saint-Denis.

NAVARro-ANDRAud Zélie

2007 Les élites urbaines de Saint-Domingue dans la seconde moitié du xv'If siècle : la place des administrateurs coloniaux (1763-1792), thèse de doctorat, Université de Toulouse, Toulouse.

Nö̈L Erik

2006

Etre Noir en France au xvII siècle, Taillandier, Paris.

OGLE Gene 2006

" "Propos injurieux" : rumor, honor and race in eighteenth century "SaintDomingue" ", in Jean Casimir, Michel Hector et Danielle Bégot (éds), La révolution et l'indépendance hä̈tiennes: autour du bicentenaire de 1804, histoire et mémoire, Bulletin de la Société d'histoire de la Guadeloupe, Gourbeyre, pp. 23-45.

Peabody Sue

1996 "There are no slaves in France 》: the political culture of race and slavery in the Ancient Regime, Oxford University Press, Oxford/New York. 


\section{Pérotin-Dumon Anne}

1998 La ville aux îles, la ville dans l'île, Basse-Terre et Pointe-à-Pitre, Guadeloupe, 1650-1815, Karthala, Paris.

Petıт Émilien

1777 Traité sur le gouvernement des esclaves, Knappen, Paris.

PLuChON Pierre

1991 Histoire de la colonisation, Fayard, Paris.

Pluchon Pierre (éd.)

$1982 \quad$ Histoire des Antilles et de la Guyane, Privat, Toulouse.

RÉGENT Frédéric

2004 Esclavage, métissage, liberté : la Guadeloupe pendant la Révolution française (1789-1802), Grasset, Paris.

ROGERs Dominique

1999 Les libres de couleur dans les capitales de Saint-Domingue: fortune, mentalités et intégration à la fin de l'Ancien Régime (1776-1789), thèse de doctorat, Université Michel de Montaigne, Bordeaux III.

2003 "De l'origine du préjugé de couleur en Haïti », in Marcel Dorigny (ed.), Haït, première république noire, SFHOM/APECE, Saint-Denis, pp. 83-101.

2006 «Entre lumières et préjugés : Moreau de Saint-Méry et les libres de couleur de Saint-Domingue », in Dominique Taffin (éd.), Moreau de Saint-Méry ou les ambiguïtés d'un Créole des Lumières, Archives départementales de la Martinique, Fort de France, pp. 77-93.

RUdDER Véronique de

1998 "Identité, origine et étiquetage, de l'ethnique au racial, savamment cultivés », Journal des anthropologues, 72 et 73, pp. 31-47.

TARRADE Jean

1991

1995 «L'esclavage est-il réformable ? Les projets des administrateurs coloniaux à la fin de l'Ancien Régime ", in Marcel Dorigny (éd.), Les abolitions de l'esclavage, de L. F. Sonthonax à V. Schalcher, 1793, 1794, 1848, Presses Universitaires de Vincennes/Éditions Unesco, Paris.

WIEIVORKa Michel

1996 «Racisme, racialisation et ethnicisation en France : cités, diversités, disparités, quelques mécanismes de ségrégation ", Hommes et migrations, 1195 , pp. 27-33.

WimprFen Alexandre Stanislas de

1993 Haïti au XVIIf siècle, richesse et esclavage dans une colonie française, Karthala, Paris. 